Int. J. Odontostomat., 10(2):177-183, 2016.

\title{
Sensibilidad y Especificidad Clínica del Indice Mandibular Canino y del Ancho Mesiodistal del Diente Canino Para Estimar el Sexo: Ajuste de un Modelo Predictivo
}

\author{
Clinical Sensitivity and Specificity of Mandibular Canine Index and Mesiodistal \\ Canine Width to Estimate Sex: Adjustment of a Predictive Model
}

\author{
${\text { Denisse Lagos*; Luis Ciocca* }{ }^{*} \text { Dante Cáceres }}^{* * *}$
}

LAGOS, D.; CIOCCA, L. \& CÁCERES, D. Sensibilidad y especificidad clínica del índice mandibular canino y del ancho mesiodistal del diente canino para estimar el sexo: ajuste de un modelo predictivo. Int. J. Odontostomat., 10(2):177-183, 2016.

RESUMEN: Los dientes son un excelente material de investigación en personas vivas y cadáveres, en el ámbito antropológico, genético, odontológico y forense, por sus características (dureza, resistencia al ataque químico, fuego y descomposición). El diente canino mandibular es el menos afectado por enfermedad periodontal, menos extraído, más resistente a traumas severos y el que presenta mayor dimorfismo sexual. Se puede usar para la estimación de sexo, calculando el Índice Mandibular Canino (IMC). El propósito de este estudio es determinar la certeza del IMC en la estimación de sexo respecto al ancho mesiodistal del canino. El estudio fue realizado en 150 sujetos $(H: M=65: 85)$, entre 18-24 años. El ancho mesiodistal del canino y la distancia canina fueron medidos en los modelos obtenidos y el IMC fue calculado. Se calculó la sensibilidad y especificidad para cada uno de los índices en cuestión. El IMC tuvo una sensibilidad del $33,85 \%$ y una especificidad de $75,29 \%$ en la estimación de sexo. El ancho mesiodistal del canino tuvo una sensibilidad de $66,15 \%$ y una especificidad de $84,71 \%$. El ancho mesiodistal del canino tiene mayor sensibilidad y especificidad que el IMC en la estimación médicolegal de sexo.

PALABRAS CLAVE: canino mandibular, ancho canino, distancia intercanina, índice mandibular canino.

\section{INTRODUCCIÓN}

La identificación médico-legal es uno de los principales procedimientos, muchas veces el primero, que se realiza para establecer la identidad de los individuos involucrados en una situación judicial, sean estos vivos o muertos. Dentro de estos últimos, los cadáveres se pueden encontrar frescos (con una data de muerte reciente), en estado de putrefacción, parcial o totalmente esqueletizados o en forma de osamentas. A su vez, el hallazgo puede ser de restos fragmentados y/o aislados o se puede hallar la totalidad del cuerpo. Dentro del proceso identificatorio, se busca establecer en primera instancia el perfil biológico del individuo, es decir, la "cuarteta básica identificatoria": una descripción cualitativa y biométrica que consiste en determinar la ancestría racial, edad, talla y sexo de él o los individuos en cuestión (Komar \& Buikstra, 2008; Senn \& Stimson, 2010; de Luca, 2011).

En lo que respecta a la determinación del sexo del individuo, cabe destacar que las diferencias sexuales entre individuos comienzan a manifestarse en el esqueleto desde antes del nacimiento: por ejemplo, la anchura de la incisura isquiática mayor de la pelvis, que es uno de los rasgos más distintivos en adultos, aumenta más rápidamente en mujeres durante el cre-

\footnotetext{
* Cirujana Dentista, Universidad de Chile. Ayudante, Área Medicina Legal Odontológica, Universidad de Chile, Santiago, Chile.

** Profesor Titular, Depto. Medicina Legal, Fac. de Medicina U. de Chile y Encargado de Área Medicina Legal Odontológica, Fac. de Odontología, U. de Chile, Santiago, Chile.

${ }^{* * *}$ Profesor Asociado, Escuela de Salud Pública, Fac. de Medicina, Universidad de Chile, Santiago, Chile.
} 
cimiento fetal. El hecho de que las mujeres crezcan y maduren más rápidamente que los varones hace necesario considerar la edad cuando se determina el sexo en individuos inmaduros, pero se puede decir que a lo largo de la infancia y la adolescencia el dimorfismo sexual se hace más marcado y es cada vez más seguro reconocer el sexo a partir de los restos esqueléticos (Ubelaker, 2007). Por encima de los 18 años de edad, las diferencias sexuales están bien definidas en el esqueleto y, si se cuenta con una buena conservación del esqueleto, se usan métodos morfo-antropológicos tradicionales, los que están basados en la observación de la pelvis y el cráneo. En general, las diferencias son en cuanto a tamaño y a forma (derivada de la función). La pelvis es considerada el elemento más dismórfico del esqueleto humano y se distingue constitutivamente de la de otros animales por su adaptación a la postura bípeda, a la contención de las vísceras pélvicas y a la locomoción (Klepinger, 2006; Black \& Ferguson, 2011; de Luca; Senn \& Stimson; Ubelaker). Dado su actuar en el trabajo de parto, la pelvis femenina es mayor en todas sus dimensiones que la masculina, aunque esta sea más robusta. Se valoran también otras estructuras como la incisura isquiática mayor (en mujeres es más ancha, formando un ángulo de $60^{\circ}$ versus un ángulo de $30^{\circ}$ en hombres), superficie auricular (más plana en hombres), surco preauricular (se presenta en mujeres y muy rara vez en hombres) acetábulo (es más grande en hombres), pubis (es más largo en mujeres y el ángulo subpúbico es mayor, caracteres relacionados con el proceso del nacimiento). La determinación de sexo a partir del cráneo, por otra parte, no es tan segura como la de la pelvis, pero puede ser usado en ausencia de ella. En el cráneo se buscan diferencias de tamaño en las inserciones musculares (del músculo esternocleidomastoideo y músculos occipitales), que suelen ser mayores en hombres; se valoran también otras estructuras como proceso mastoides, glabela, líneas nucales, protuberancia occipital externa y mandíbula (ángulo mandibular y protuberancia mental, particularmente). Cuando el esqueleto está pobremente conservado o se dispone de estructuras aisladas, cobra importancia el uso de algunas funciones discriminantes, con mediciones de algunos huesos largos representativos (húmero, fémur, tibia) (de Luca; Klepinger; Ubelaker).

Sin embargo, muchas veces el investigador se encuentra ante la presencia de osamentas y/o restos cadavéricos, partes de cuerpos, cuerpos carbonizados o en un estado de descomposición avanzado. En estos casos cumple un rol fundamental la identificación por métodos odontológicos. De hecho, la INTERPOL describe el análisis odontológico forense como uno de los métodos de identificación primario en caso de catástrofes, en conjunto con el análisis de las huellas dactilares y el de DNA (Interpol, 2009). Dentro de las ventajas que ofrece la identificación por métodos odontológicos se encuentra la gran cantidad de características individualizantes (32 dientes, con 5 superficies cada uno, sumado a las restauraciones y su disposición dentro del arco), el hecho de que los dientes poseen el tejido más duro del organismo humano y que estén protegidos dentro de la cavidad oral, siendo resistentes a diversas noxas como ataque químico, fuego y descomposición, los hacen elementos idóneos para la labor identificatoria (Ciocca Gómez, 2009; Black \& Ferguson; Interpol). De la misma manera, son también resistentes a algunos agentes externos los materiales ocupados en su restauración. Dentro de las pocas desventajas que presenta la identificación basada en estos métodos están la ausencia de registros de atención ante-mortem, la alteración de registros o confusión en su interpretación, pues no existe acuerdo en el gremio respecto a la nomenclatura usada (Rodríguez Cuenca, 2003; Avon, 2004; Blakaj et al., 2010; Black \& Ferguson; Ciocca Gómez).

La ABFO (American Board of Forensic Odontology, 2012) agrupa en tres grandes grupos los métodos de identificación odontológicos para estimar sexo: la presencia de cuerpos de Barr, la Amelogenina y la morfología de dientes y estructuras maxilofaciales. Los cuerpos cromáticos de Barr son una condensación de la cromatina presentes en el núcleo de las células en el sexo femenino, se puede observar en distintos tipos celulares (células pulpares, óseas, de la retina y de la mucosa oral) (Suazo et al., 2010). La amelogenina, por su parte, es una proteína codificada en los cromosomas sexuales y tiene una distinta secuencia y tamaño entre los alelos codificados en el cromosoma $X$ y el cromosoma $Y$ (AMELX y AMELY, respectivamente) (Frances et al., 2008). Respecto al último punto, morfología de dientes y estructuras maxilofaciales, hay varias relaciones descritas en la literatura: los dientes de los hombres son, en general, mayor en todas sus dimensiones que los de las mujeres; la relación del ancho mesiodistal entre el incisivo central y el lateral es menor en la mujer, es decir, sus dientes son más uniformes que en el hombre; el diámetro buco-lingual en premolares y molares sería mayor en hombres, etc. (Moya Pueyo et al., 2004; Condon et al., 2011; Ciocca Gómez). Dentro de las estructuras maxilofaciales, reviste especial importancia el hueso mandibular, especialmente cuando se trata de la identificación de individuos subadultos: características como el pronunciamiento del mentón (que en 
los varones se presenta prominente y cuadrangular, con estructuras elevadas y rugosas, al contrario de las niñas), la forma del arco dental anterior (que es más ancho en niños, con los alvéolos de los caninos que sobresalen, determinando una forma de $U$ ) y la región goníaca (que en el caso de los niños se encuentra evertida, ligeramente sobresaliente en una vista superior) (Rodríguez Cuenca).

Particularmente, para la determinación del sexo, es sabido que los dientes caninos inferiores son los que presentan mayor dimorfismo, incluso se describe un índice de dimorfismo sexual (IDS) mayor que el resto de los dientes, alcanzando valores de 1,08 y 1,09 (Rodríguez Cuenca). Además de esta característica, son también los dientes menos afectados por enfermedad periodontal, los menos extraídos y los más resistentes a traumas severos (Kaushal et al., 2003; Padmavati et al., 2011). Esta diferencia es explicada por la actividad del canino, ya que éste, a diferencia del resto de los dientes, no tiene una función masticatoria, sino que evolutivamente se le atribuye una función de agresión y defensa. Es esta diferencia la que ha sido propuesta para su uso en identificación forense (Kaushal et al., 2003, 2004; Mughal et al., 2010; Aggarwal et al., 2010). Rao et al. (1989) propusieron el "Índice Mandibular Canino" como un método válido para la determinación de sexo que requiere para su cálculo algunas medidas tomadas a partir de los dientes caninos mandibulares:

IMC= Ancho mesio-distal de la corona del canino / Distancia intercanina

El propósito de este estudio fue determinar la certeza del IMC en la estimación de sexo (usando valor de 0,274 obtenido por Rao et al., como valor de corte) versus el Ancho Mesiodistal del Canino por sí mismo, al aplicarlo en un grupo de individuos chilenos.

\section{MATERIAL Y MÉTODO}

El estudio fue hecho en una muestra constituida por 150 sujetos, 65 hombres y 85 mujeres, pacientes y alumnos de la Facultad de Odontología de la U. de Chile y de pacientes de Clínicas privadas, con un rango de edad entre 18 y 24 años.

Se consideraron criterios de inclusión: salud periodontal y gingival, caninos mandibulares libres de caries, overbite y overjet de 2 a $3 \mathrm{~mm}$, ausencia de espacio entre dientes anteroinferiores y relación molar y canina normal (clase I). Mientras que los criterios de exclusión fueron presencia de apiñamiento dentario en dientes anteroinferiores y la presencia de facetas de desgaste incisal a nivel de caninos mandibulares. Cabe destacar, que los participantes Ilenaron una ficha de datos respecto a su edad y ascendencia étnica.

Previa firma de consentimiento informado, se procedió a tomar una impresión de la mandíbula con alginato tipo 2, la que fue vaciada en yeso odontológico tipo IV para evitar contracciones y obtener una mejor superficie. Con un pie de metro (calibrador) digital marca RedLine Mechanics ${ }^{\circledR}$, precisión $0,01 \mathrm{~mm}$, se tomaron las siguientes medidas:

- Medición del ancho mesiodistal de los caninos mandibulares: fue considerado como el mayor ancho del diente canino medido entre sus puntos de contacto con los dientes vecinos.

- Medición de la distancia intercanina: fue medida entre las cúspides de los caninos mandibulares derecho e izquierdo.

- Cálculo de Índice Mandibular Canino: el IMC es una razón entre dos parámetros medidos en los caninos mandibulares, según la siguiente fórmula:

IMC= Diámetro mesiodistal de la corona del canino / Ancho del arco mandibular entre los caninos

Plan de análisis. Todos los datos obtenidos de las muestras fueron ordenados en planilla de cálculo Microsoft Office Excel $2007^{\circledR}$ y procesados mediante los softwares estadísticos Epidat $4.0^{\circledR}$ y Stata $11.0^{\circledR}$.

Se calculó la sensibilidad y especificidad del Índice Mandibular Canino (IMC) para predecir sexo, considerando como punto de corte el valor de IMC establecido por Rao et al., a saber: 0,274.

Para el análisis del Ancho Mesiodistal del Canino, a partir de las mediciones hechas, se ajustó un modelo predictivo a través de una regresión logística cuya variable respuesta es el sexo, ajustando por la variable explicativa "ancho mesiodistal del canino" (considerando el mayor entre el canino derecho y el izquierdo). Una vez obtenida la fórmula respectiva, se calculó la sensibilidad y especificidad para el ancho mesiodistal del canino.

Se graficó en curvas ROC la sensibilidad y especificidad del IMC y del ancho mesiodistal del canino, respectivamente. Las curvas ROC (receiver 
operating charasteristic curve) ilustran de manera gráfica la sensibilidad y la especificidad de un método diagnóstico. Uno de sus propósitos específicos es determinar la capacidad discriminativa de un test diagnóstico. Para comparar la capacidad discriminativa de dos test, se comparan las áreas bajo la curva de cada curva ROC, siendo la prueba con mayor capacidad discriminativa la prueba cuya curva ROC tenga mayor área bajo la curva.

Fue considerado como un método certero en la estimación de sexo, aquél que presentó una sensibilidad y especificidad mayor al $90 \%$.

\section{RESULTADOS}

Sensibilidad y especificidad del IMC. En la aplicación del IMC para la determinación del sexo se consideró un punto de corte de 0,274 , tal como fue propuesto por Rao et al. en 1989, valor por encima del cual se consideró que el sujeto era hombre, mientras que valores inferiores fueron considerados sujetos mujeres. De esta manera, se aprecia que 22 sujetos fueron correctamente clasificados como hombres y 64 fueron correctamente clasificados como mujeres (Tabla I). Por lo tanto, el IMC tiene una sensibilidad de $33,85 \%$ y una especificidad de $75,29 \%$ en la determinación de sexo.

La curva ROC correspondiente al análisis del IMC presenta un área bajo la curva de 0,5457 , muy cercana a la diagonal de referencia (Fig. 1).

Sensibilidad y especificidad del ancho mesiodistal del canino. De los datos obtenidos, se obtuvo la siguiente función logística que explica el comportamiento del Ancho Mesiodistal del Canino: $y=1 / 1+e-$ $(3,860284 x-27,95429)$, donde " $x$ " corresponde al ancho mesiodistal del canino medido en milímetros, e "y" al sexo del individuo (valores entre 0 y 0,5 fueron considerados mujeres, mientras que valores sobre 0,5 fueron considerados hombres). De esta manera, fueron correctamente clasificados 43 hombres y $72 \mathrm{mu}$ jeres, como se muestra en la Tabla II. Por lo tanto, el Ancho Mesiodistal del Canino tiene una sensibilidad de un $66,15 \%$ y una especificidad de un $84,71 \%$.

La curva ROC correspondiente al análisis del Ancho Mesiodistal del Canino presenta un área bajo la curva de 0,8524 , alejándose de la diagonal de referencia y acercándose a 1 (Fig. 2).
Todas las mediciones tienen un Coeficiente de Correlación Intraclase (ICC) mayor a 0,9 los que nos indica mediciones altamente confiables y reproducibles.

Tabla I. Clasificación por sexo de los sujetos de la muestra según el Índice Mandibular Canino calculado.

\begin{tabular}{lccc}
\hline \multirow{2}{*}{ Clasificación de sexo } & \multicolumn{2}{c}{ Sexo } & Total \\
\cline { 2 - 3 } & Hombre & Mujer & \\
\hline Hombre $>0,274$ & 22 & 21 & 43 \\
Mujer $\leq 0,274$ & 43 & 64 & 107 \\
Total & 65 & 85 & 150 \\
\hline
\end{tabular}

Tabla II. Clasificación por sexo de los sujetos de la muestra según función logística dependiente del ancho mesiodistal del diente canino.

\begin{tabular}{lccc}
\hline \multirow{2}{*}{ Clasificación de sexo } & \multicolumn{2}{c}{ Sexo } & \multirow{2}{*}{ Total } \\
\cline { 2 - 3 } & Hombre & Mujer & \\
\hline Hombre & 43 & 13 & 56 \\
Mujer & 22 & 72 & 94 \\
Total & 65 & 85 & 150 \\
\hline
\end{tabular}

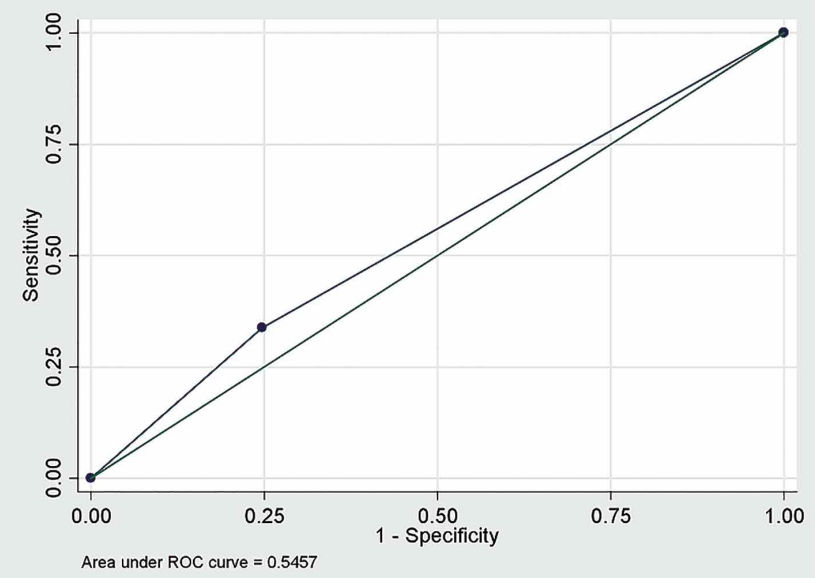

Fig. 1. Curva ROC del Índice Mandibular Canino.

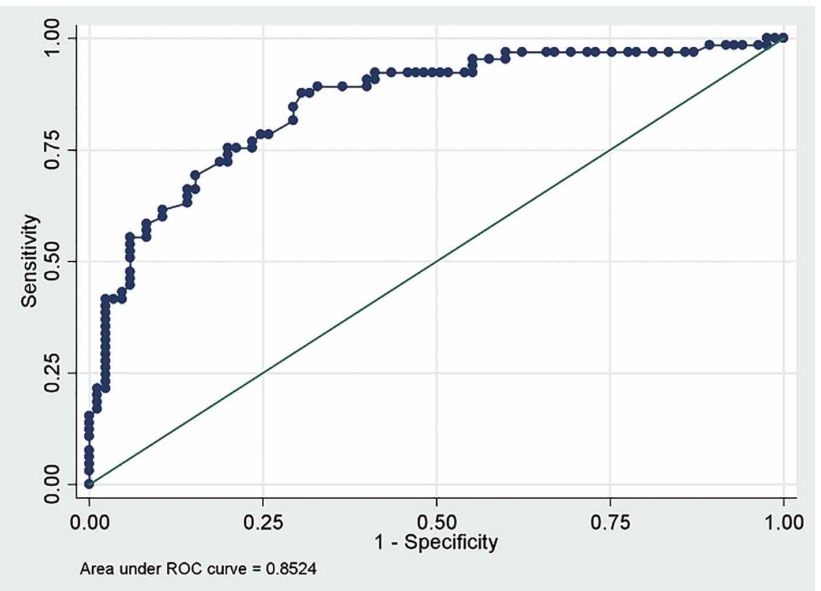

Fig. 2. Curva ROC del ancho mesiodistal del diente canino. 


\section{DISCUSIÓN}

En la mayoría de los primates, la función de los caninos mandibulares no es masticatoria, sino que, sobre una base evolutiva, está ligada a la defensa y a la agresión, por lo tanto, a la supervivencia. Eimerl \& DeVore (1965) postularon que, en la evolución de los primates, hubo una transferencia de esta función desde los caninos en los monos a los dedos de las manos y pies en el hombre y, hasta que esta transferencia se complete, la supervivencia es dependiente de los caninos, especialmente en los machos (Aggarwal et al.; Kaushal et al., 2003, 2004; Mughal et al.). Ayudan también a esta disminución del porcentaje de dimorfismo sexual el proceso de sedentarización en la época contemporánea, especialmente en algunas estructuras como la talla y las dimensiones del cráneo y dientes (Rodríguez Cuenca). No es coincidencia, por tanto, que los caninos inferiores sean los dientes que muestren mayor dimorfismo sexual y sean los dientes más resistentes a enfermedad periodontal y traumas y sean también los menos afectados por caries (Kaushal et al., 2003; Padmavati et al.).

En 1989, Rao et al. señalaron que el canino mandibular es el diente con más resistencia a la enfermedad periodontal y los traumas, por lo que su estudio es fundamental para la determinación de sexo por métodos odontológicos. Establecen el Índice Mandibular Canino, clasificando correctamente a un $84,3 \%$ de los hombres y a un $87,5 \%$ de las mujeres de su muestra de 382 hombres y 384 mujeres (Rao et al.). En otros estudios similares a los de este autor, se ha demostrado la validez de este índice (Kaushal et al., 2004; Reddy et al., 2008; Boaz \& Gupta, 2009; Ahuja \& Manchanda, 2011; Aggarwal et al.; Mugal et al.; Padmavati et al.), sin embargo, otros estudios no han ratificado al IMC como instrumento válido para la estimación de sexo, a diferencia del Ancho Mesiodistal del Canino, que sí lo hace de forma significativa (Acharya \& Mainali, 2009; Acharya et al., 2011; Sharma \& Gorea, 2010). Los resultados obtenidos en el presente estudio coinciden con estos últimos autores e indican que el Ancho Mesiodistal del Canino tiene mayor capacidad discriminativa que el IMC, esto es evidenciado en una mayor sensibilidad y especificidad del ancho mesiodistal del canino respecto al IMC $(33,85 \%$ y $75,29 \%$ vs. un $66,15 \%$ y $84,71 \%$, respectivamente) y al comparar las áreas bajo la curva de las respectivas curvas $\operatorname{ROC}(0,5457$ vs. 0,8524$)$.
Esto tiene implicancias prácticas: el IMC requiere para su aplicación de sujetos que cumplan con criterios de inclusión muchas veces difíciles de encontrar en la mayor parte de la población, ya sea por falta de higiene, presencia de hábitos parafuncionales como el bruxismo, pobre acceso a programas de salud oral, etc. y hacen que este índice sea aplicable a un grupo muy acotado de la población. En tanto que para la aplicación de un test que tenga como variable única el ancho mesiodistal del canino podemos obviar varios de estos criterios, ya que podemos aplicarlo en individuos que presenten problemas periodontales, que hayan sido sometidos a tratamiento de ortodoncia, que presenten desgaste incisal, que tengan apiñamiento o espacios en la zona anteroinferior, indistintamente. Incluso, cuando no se cuente con el hueso mandibular completo o una sección de él, el método se podría aplicar sobre dientes caninos mandibulares aislados, lo que facilita la labor identificatoria. De esta manera, el Ancho Mesiodistal del canino emerge como un método con mayores ventajas, dado que requiere solamente una medición que involucra exclusivamente a dicho diente.

Sin embargo, los resultados deben ser tomados con cautela, porque, si bien la capacidad discriminativa del ancho mesiodistal del canino es mejor que la del IMC, estos resultados no permiten enunciarlo como un método de alto poder discriminante en la determinación del sexo por sí solo, dado que no fue alcanzado el $90 \%$ de aciertos propuesto en la metodología. Esta baja sensibilidad y especificidad podría deberse al proceso natural de evolución, donde se describe una reducción del dimorfismo sexual, especialmente en aquellas características ligadas a la supervivencia, como es el caso del canino mandibular, llevando a similitudes en el ancho canino en ambos sexos. También, según diversos autores, es de gran importancia considerar en los estudios que implican variables antropométricas, la variable "ascendencia étnica-raza". Esta variable pudiera estar determinando diferencias entre hombres y mujeres y, por lo tanto, entre poblaciones. Particularmente, en nuestro país se presenta una distribución étnica que considera un componente caucásico en menor medida y otro componente mayormente amerindio (Valenzuela, 2002; Venegas et al., 2008). Finalmente, el Ancho Mesiodistal del Canino es un método simple, de bajo costo y de rápida ejecución, por lo tanto, muy útil en la práctica forense habitual. 
LAGOS, D.; CIOCCA, L. \& CÁCERES, D. Clinical sensitivity and specificity of mandibular canine index and of mesiodistal canine width to estimate sex: adjustment of a predictive model. Int. J. Odontostomat., 10(2):177183, 2016.

ABSTRACT: Teeth are an excellent research material in living persons and in corpses, in the anthropological, genetic, dental and forensic fields, because of their characteristics (hardness, resistance to chemical attack, fire and decay). Mandibular canine teeth are the least affected by periodontal disease, the least extracted teeth, are more resistant to severe trauma and have a greater sexual dimorphism. They can be used for sex estimation, through the Mandibular Canine Index (MCl). The purpose of this study is to determine the accuracy of the $\mathrm{MCl}$ in sex estimation, compared to the mesiodistal canine width method. The study was conducted on 150 subjects $(M: F=65: 85)$, aged 18-24. The mesiodistal width of canine and the intercanine distance were measured on the obtained models, and the $\mathrm{MCl}$ was calculated. The sensitivity and specificity for each of the two indexes were also calculated. $\mathrm{MCl}$ had a sensitivity of $33.85 \%$ and a specificity of $75.29 \%$ in sex estimation. The mesiodistal width of canine had a sensitivity of $66.15 \%$ and a specificity of $84.71 \%$. The mesiodistal width of canine has a higher sensitivity and specificity than the $\mathrm{MCl}$ in the medicolegal estimation of sex.

KEY WORDS: mandibular canine, canine width, intercanine distance, mandibular canine index.

\section{REFERENCIAS BIBLIOGRÁFICAS}

American Board of Forensic Odontology, Inc. Diplomates Reference Manual. 2013. Disponible en: http:// www.abfo.org/wp-content/uploads/2012/08/ABFOReference-Manual-1-22-2013-revision.pdf

Acharya, A. B.; Angadi, P. V.; Prabhu, S. \& Nagnur, S. Validity of the mandibular canine index $(\mathrm{MCl})$ in sex prediction: Reassessment in an Indian sample. Forensic Sci. Int., 204(1-3):207.e1-4, 2011.

Acharya, A. B. \& Mainali, S. Limitations of the mandibular canine index in sex assessment. J. Forensic Leg. Med., 16(2):67-9, 2009.

Aggarwal, B.; Kaushal, S.; Vasudeva, K.; Chhabra, U. \& Singla, S. Significance of mandibular canine index in sexual dimorphism. J. Indo Pac. Acad. Forensic Odontol., 1(1):1-4, 2010.

Ahuja, P. \& Manchanda, A. Application of oral hard and soft tissue structures in sex determination. Internet $J$. Forensic Sci., 4(2), 2011.

Avon, S. L. Forensic odontology: the roles and responsibilities of the dentist. J. Can. Dent. Assoc., 70(7):453-8, 2004.

Blakaj, F.; Bicaj, T. \& Bicaj, B. Dental identification of a decomposed body. Med. Arh., 64(2):125-6, 2010.

Boaz, K. \& Gupta, C. Dimorphism in human maxillary and madibular canines in establishment of gender. $J$. Forensic Sci., 1(1):42-4, 2009.
Black, S. \& Ferguson, E. Forensic Anthropology: 2000 to 2010. Boca Raton, CRS Press, 2011.

Ciocca Gómez, L. Odontología Médico-Legal. Aspectos Forenses, Profesionales y Sociales. Santiago, Ed. Jurídicas de Santiago, 2010.

Condon, M.; Bready, M.; Quinn, F.; O'Connell, B. C.; Houston, F. J. \& O'Sullivan, M. Maxillary anterior tooth dimensions and proportions in an Irish young adult population. J. Oral Rehabil., 38(7):501-8, 2011.

de Luca, S. Identificación Humana en Antropología Forense: Aportaciones para la Estimación de Sexo y Edad. Tesis Doctoral. Granada, Universidad de Granada, 2011.

Eimerl, S. \& DeVore, I. The Primates. Life Nature Library. New York, Time-Life International, 1965.

Francès, F.; Castelló, A. \& Verdú, F. El diagnóstico genético del sexo mediante el test de la amelogenina: Métodos y posibles fuentes de error. Cuad. Med. Forense, 14(52):119-25, 2008.

Interpol. Guía para la Identificación de Víctimas de Catástrofes. 2009. Disponible en: http://www.interpol.int/ content/download/10461/74528/version/4/file/ guide[1].pdf

Kaushal, S.; Patnaik, V. V. G. \& Agnihotri, G. Mandibular canines in sex determination. J. Anat. Soc. India., 52(2):119-24, 2003. 
Kaushal, S.; Patnaik, V. V. G.; Sood, V. \& Agnihotri, G. Sex determination in north Indians using mandibular canine index. J. Indian Acad. Forensic Med., 26(2):45-9, 2004.

Klepinger, L. L. Fundamentals of Forensic Anthropology. Hoboken, Wiley-Liss, Publication, 2006.

Komar, D. \& Buikstra, J. Forensic Anthropology. Contemporary Theory and Practice. New York, Oxford University Press, 2007.

Moya Pueyo, V.; Roldán Garrido, B. \& Sánchez Sánchez, J. A. Odontología Legal y Forense. Madrid, Masson, 1994.

Mughal, I. A.; Saqib, A. S. \& Manzur, F. Mandibular canine index $(\mathrm{MCl})$; its role in determining gender. Prof. Med. J., 17(3):459-63, 2010.

Rao, N. G.; Rao, N. N.; Pai, M. L. \& Kotian, M. S. Mandibular canine index--a clue for establishing sex identity. Forensic Sci. Int., 42(3):249-54, 1989.

Padmavati, K.; Farah, V. M.; Syed, A. A. \& Ather, S. A. Mandibular canine index: A tool for sex determination. J. Indian Dent. Assoc., 5(1):18-20, 2011.

Reddy, V. M.; Saxena, S. \& Bansal, P. Mandibular canine index as a sex determinant: A study on the population of western Uttar Pradesh. J. Oral Maxillofac. Pathol., 12(2):56-9, 2008.

Rodríguez Cuenca, J. V. Dientes y Diversidad Humana: Avances de la Antropología Dental. Bogotá, Editora Guadalupe Ltda., 2003.

Sharma, M.; Gorea, R.K. Importance of mandibular and maxillary canines in sex determination. J. Punjab. Acad. Forensic Med. Toxicol., 10:27-30, 2010.

Senn, D. R. \& Stimson, P. G. Forensic Dentistry. 2a ed. Boca Raton, CRS Press, 2010.

Suazo, G. I.; Roa, H. I. \& Cantín L., M. Sex chromatin in dental pulp: Performance of diagnosis test and gold standard generation. Int. J. Morphol., 28(4):1093-6, 2010.

Ubelaker, D. H. Enterramientos Humanos: Excavación, Análisis, Interpretación. Donostia, Sociedad de Ciencias Aranzadi, 2007.

Valenzuela Y., C. El Gradiente Sociogenético Chileno y sus Implicaciones Ético-Sociales. Santiago de Chile, Medwave Ciencia, 2002.

Vanegas L., J.; Villalón C., M. \& Valenzuela Y., C. Consideraciones acerca del uso de la variable etnia/raza en investigación epidemiológica para la Salud Pública: A propósito de investigaciones en inequidades. Rev. Med. Chile, 136(5):637-44, 2008.

\author{
Dirección para Correspondencia: \\ Dra. Denisse Lagos Tissie \\ Cirujana Dentista \\ Área Medicina Legal Odontológica \\ Universidad de Chile \\ Santiago \\ CHILE
}

Teléfono: (+56-2) 29781737

Email: dlagos@odontologia.uchile.cl

Recibido: 11-01-2016

Aceptado: 14-04-2016 